\title{
Intervenciones en estrés laboral: un análisis a partir del modelo bioecológico de Bronfenbrenner
}

\author{
Workplace stress interventions: An analysis \\ based on the Bronfenbrenner's bio-ecological model
}

\author{
Angélica María Hermosa-Rodríguez ${ }^{1}$ \\ Citación: Hermosa R., A.M. (2019). Intervenciones en estrés laboral: un análisis a partir del modelo \\ bioecológico de Bronfenbrenner. Psicología y Salud, 29(2), 167-176.
}

RESUMEN

\begin{abstract}
Este trabajo tuvo como objetivo analizar las intervenciones en el estrés laboral a la luz de los fundamentos conceptuales y metodológicos del modelo bioecológico de Urie Bronfenbrenner. Dicho modelo ofrece un marco de referencia que trasciende los ámbitos individual y organizacional bajo los cuales se ha intervenido tradicionalmente en el problema. Se hace una contextualización del estrés laboral y las intervenciones en este campo; luego se describe la perspectiva teórico-conceptual y los aspectos metodológicos del citado modelo, y finalmente se toman sus postulados como una propuesta para orientar las intervenciones en el estrés laboral.
\end{abstract}

Palabras clave: Estrés laboral; Salud ocupacional; Intervenciones en estrés laboral; Modelo bioecológico.

\begin{abstract}
The present paper examines workplace stress interventions in the context of the conceptual and methodological proposals of Bronfenbrenner's bio-ecological model. This model provides a framework that transcends the individual and organizational levels under which work stress has been traditionally conceptualized and intervened. The paper begins with a contextualization of work stress and interventions in this field, followed by a description of the theoretical-conceptual perspectives and methodological aspects of the model and it finally proposes the model as a source to address and develop workplace stress interventions.
\end{abstract}

Key words: Work stress; Occupational health; Job stress interventions; Bio-ecological model.

\section{INTRODUCCIÓN}

$\mathrm{E}$ n un mundo globalizado como el actual, ciertas situaciones sociales y económicas, como los altibajos financieros, la introducción de nuevas tecnologías y la desregulación de los mercados laborales, afectan la economía de los países, la organización del trabajo y la relación hombre-trabajo. Estas situaciones, sumadas a factores tales como la intensificación del trabajo (v.g. incremento del ritmo y la carga laboral) y la precariedad del trabajo (v.g. condiciones laborales inestables), favorecen la aparición del estrés laboral, reconocido por la Organización Internacional del Trabajo (OIT) (2016) como “...un problema global que afecta a todas las profesiones y los trabajadores tanto de países en desarrollo como de los desarrollados" (p. 2).

\footnotetext{
${ }^{1}$ Departamento de Administración de Empresas, Universidad Central, Calle 21 Núm. 4-40, Bogotá, Colombia, tel. (057)323-98-68, ext. 3753, correo electrónico: ahermosar@ucentral.edu.co. Artículo recibido el 24 de abril y aceptado el 16 de mayo de 2018.
} 
Un estudio realizado por la propia OIT (2010) sobre riesgos emergentes y nuevos modelos de prevención señala que el estrés es la segunda causa registrada de los trastornos de la salud relacionados con el trabajo en la Unión Europea. Asimismo, dicho organismo multilateral ha alertado sobre la relación que existe entre el estrés laboral y los trastornos del sistema osteomuscular, la hipertensión, las úlceras pépticas, las enfermedades cardiovasculares, los trastornos del estado de ánimo (v.g. depresión y ansiedad) e incluso el suicidio. Tales hechos son tan graves que los trastornos de salud mental relacionados con el estrés laboral son la principal causa de muerte prematura en Europa (Kortum, Leka y Cox, 2010).

En países con economías emergentes, como Colombia, la OIT (2016) reconoce avances en la investigación del estrés laboral y de los agentes de riesgo que lo pueden causar. En este país, el Ministerio de la Protección Social (2007) desarrolló la I Encuesta Nacional de Condiciones de Salud y Trabajo en el Sistema General de Riesgos Profesionales, en el que se evidenció que entre $20 \mathrm{y}$ $33 \%$ de los trabajadores encuestados manifestaron sentir altos niveles de estrés. De igual manera, los factores psicosociales estuvieron relacionados entre 6 y $15 \%$ con la ocurrencia de accidentes de trabajo. Ante estos resultados, en el mismo documento se recomienda "establecer programas o líneas de intervención y control de factores de riesgo psicosocial..." (p. 107) y se sugiere "privilegiar intervenciones preventivas de accidentes de trabajo [...] donde este evento es más frecuente y letal" (p. 107).

La II Encuesta Nacional de Condiciones de Seguridad y Salud en el Trabajo del Sistema General de Riesgos Laborales y Trabajo realizada por el Ministerio de Trabajo colombiano (2015) evidenció que los riesgos de orden psicosocial continúan siendo muy frecuentes en la población trabajadora; por ejemplo, responsabilidades laborales que no están claramente definidas $(74 \%)$ y atención directa al público (67\%). Asimismo, se han hecho estudios sobre el nivel de estrés o programas para su control tan solo en $14 \%$ de las empresas encuestadas. A pesar de ese bajo porcentaje, hay un creciente interés por parte de las empresas por trabajar más aún en el diagnóstico y la intervención del estrés entre los trabajadores.
Tal como lo afirma la OIT (2016), todos los grupos ocupacionales pueden verse afectados por el estrés laboral; por tal razón, los profesionales y los investigadores encargados de estudiar este fenómeno están obligados a profundizar en su etiología, en las condiciones psicosociales en que se realiza el trabajo, en las posibles consecuencias para la salud del individuo y en el diseño e implementación de intervenciones que busquen mejorar la salud de los trabajadores. Es sobre esto que el presente artículo pretende alertar.

La literatura en este campo da cuenta de un cúmulo importante de estudios sobre programas de intervención en el estrés laboral centrados primordialmente en estrategias de intervención de tipo individual (Hahn, Binnewies, Sonnentag y Mojza, 2011; Maes y Boersma, 2005; Nielsen, Randall, Holten y Rial-Gonzalez, 2010; Nielsen, Taris y Cox, 2010; Unsworth y Mason, 2012). Sin embargo, Lamontagne, Keegel, Louie, Ostry y Landsbergis (2007) hallaron que existe una proporción creciente de intervenciones enfocadas simultáneamente en dos niveles: organizacional e individual. Tal resultado indica un interés progresivo en el diseño de intervenciones desde una perspectiva multinivel; estudios recientes reclaman que la investigación sobre el estrés relacionado con el trabajo trascienda el ambiente laboral y se incluya en el contexto social, político y económico de los países (Kortum et al., 2010).

El diseño e implementación de intervenciones con las características antes mencionadas debe fundamentarse en un corpus teórico-empírico robusto (Maes y Boersma, 2005). Orleans (2008) asevera que el intercambio dinámico entre teoría, investigación y práctica es crítico si se aspira a promover de manera efectiva la salud en diferentes espacios, como el laboral, entre otros. De ahí que usar teorías en el diseño, instrumentación y evaluación de intervenciones da como resultado que estas sean más potentes y se afirmen las teorías. Glanz, Rimer y Viswanath (2008) aseguran que se pueden diseñar mejores intervenciones cuando se comprenden las teorías y se tiene la capacidad de utilizarlas con destreza en la investigación y en la práctica.

Así, analizar las intervenciones en el área del estrés laboral a partir de modelos explicativos que la psicología de la salud ocupacional no ha 
considerado aún puede ampliar el conocimiento que se tiene sobre este campo en particular.

El presente artículo pretende evidenciar que el modelo bioecológico del desarrollo humano ofrece una serie de elementos conceptuales y metodológicos que hacen posible el abordaje de estas intervenciones desde una perspectiva multinivel. Dicho modelo, conocido como el modelo proceso-persona-contexto-tiempo (РPCT) (Bronfenbrenner, 2001), parte del supuesto de que cada persona se encuentra involucrada en una serie de niveles relacionales (individual, familiar, comunitario y social) y sugiere un diseño de investigación que facilite el estudio de los mismos. Con este modelo es posible examinar las bases culturales e institucionales que subyacen al surgimiento y mantenimiento del estrés laboral como una de las modalidades del malestar psicosocial en el trabajo. De manera similar, proporciona una perspectiva holística de la salud en el trabajo porque no se limita a la intervención del fenómeno considerando meramente al individuo o a la organización, sino que trasciende esos límites y los complementa con el marco extralaboral.

En este orden de ideas, diferentes autores afirman que en el diseño, implementación y evaluación de intervenciones sobre el estrés laboral no se puede ignorar el contexto cultural, político, económico y social, pues no considerar dichos factores puede afectar la efectividad de aquéllas (Brough y O'Driscoll, 2005; Hahn et al., 2011; Hansson, Vingard, Arnetz y Anderzén, 2008; Henry, 2005; Nielsen, Randall et al., 2010; Nielsen, Taris y Cox, 2010; Unsworth y Mason, 2012). Este hecho es confirmado por Cox, Taris y Nielsen (2010), quienes señalan que a pesar de que se están diseñando continuamente nuevas intervenciones, al parecer no corresponden a la necesidad social de intervenciones útiles y efectivas en la práctica; es decir, el foco de las intervenciones debe radicar en planteamientos innovadores que mejoren la salud de los trabajadores y su bienestar. Así, el modelo bioecológico de Bronfenbrenner ofrece explicaciones plausibles para dar mayor eficacia a las intervenciones en el área del estrés laboral porque permite pensar en un modelo de intervención que promueve el bienestar y previene el malestar en el trabajo, apoyado en la transformación de las dinámicas sociales, institucionales, económicas y culturales.

\section{El modelo bioecológico de Bronfenbrenner y las intervenciones en el estrés laboral}

La perspectiva teórica y metodológica del modelo de Urie Bronfenbrenner destaca la importancia que tiene el modo en que la persona percibe el ambiente que le rodea y la forma en que se relaciona con él. Para este autor, el ambiente que circunscribe al sujeto es la principal fuente de influencia sobre su conducta y es entendido como ambiente ecológico.

El modelo bioecológico tiene propiedades distintivas que Bronfenbrenner ha expresado en forma de proposiciones. La primera de ellas plantea que la experiencia es un elemento clave, pues cualquier ambiente relacionado con el desarrollo humano incluye no solo las propiedades objetivas del mismo, sino también la manera en que estas propiedades son experimentadas subjetivamente por las personas y modificadas por ellas durante el curso de su vida (Bronfenbrenner, 2001). El autor agrega que los elementos objetivos y subjetivos son complementarios: ninguno se presume de manera aislada como suficiente para explicar el curso del desarrollo humano.

Si se aplica este principio al contexto laboral, los trabajadores están en permanente relación con las propiedades objetivas del ambiente de trabajo y con su propia subjetividad (e.g. creencias personales, emociones, actitudes). Sin embargo, muchas de las intervenciones en el estrés laboral carecen de esta visión complementaria, lo que se revela en aspectos metodológicos tales como las técnicas de recolección de datos que se emplean en este tipo de estudios. En la mayoría de los casos, son instrumentos de autorreporte que evalúan la percepción del trabajador sobre las condiciones del ambiente laboral, su propia salud, las características individuales o el estrés percibido, entre otros (Brinkborg, Michanek, Hesser y Berglund, 2011; Hahn et al., 2011; Hatinen, Kinnunen, Pekkonen y Kalimo, 2007; Le Blanc, Hox, Schaufeli, Taris y Peeters, 2007; Orly, Rivka, Rivka y Dorit, 2012; Unsworth y Mason, 2012), y poco se acude a la combinación de medidas objetivas y subjetivas (Wolever et al., 2012).

La segunda proposición contiene un componente teórico importante del modelo: los procesos proximales, definidos como aquellos procesos progresivamente complejos de interacción recíproca entre un organismo humano biopsicológico activo 
que evoluciona, y las personas, objetos y símbolos de su ambiente externo inmediato; tal interacción debe ocurrir a lo largo de periodos prolongados para que sea efectiva (Bronfenbrenner, 2001; Bronfenbrenner y Morris, 2006). En el ámbito laboral, los procesos proximales se expresan de diferentes formas duraderas de interacción, como las relaciones con el jefe, los colegas y los compañeros de trabajo, y el aprendizaje de nuevas habilidades o conocimientos, entre otros. Esta interacción progresiva se ha evaluado en diversas intervenciones en esta área, especialmente la relación entre el trabajador y sus jefes, supervisores y compañeros de trabajo (Hatinen et al., 2007; Le Blanc et al., 2007). También se ha analizado la adquisición de conocimientos, el aprendizaje de nuevas habilidades para el trabajo y el modo en que estos procesos pueden disminuir el estrés laboral (Andersson-Felé, 2005).

En la tercera proposición se plantean aspectos metodológicos del modelo bioecológico; concretamente, las estrategias que permiten dar respuesta a los interrogantes de investigación. Los procesos proximales varían sistemáticamente en función de las características de la persona, el contexto ambiental (inmediato y más remoto) en el que los procesos tienen lugar o las continuidades y cambios que ocurren en ese ambiente a través del tiempo. El planteamiento citado se traduce en un diseño de investigación denominado Modelo proceso-persona-contexto-tiempo (PPCT en lo sucesivo) (Bronfenbrenner, 2001; Bronfenbrenner y Morris, 2006). En otras palabras, las investigaciones en el campo del desarrollo deben estudiar simultáneamente los procesos proximales y las características de la persona y del contexto y las relacionadas con el tiempo. Aunado a ello, se deben ejecutar análisis estadísticos que permitan estudiar las interacciones más relevantes entre estos múltiples factores (Guhn y Goelman, 2011). Aunque Bronfenbrenner ha manifestado que el modelo PPCT es muy ambicioso para utilizarse en las investigaciones porque no muchas estarían en condiciones de examinar todas las interacciones entre los diferentes componentes del modelo, hay estudios en el campo del desarrollo infantil que han indagado esas interacciones (Bronfenbrenner, 2001; Guhn y Goelman, 2011; Reiss, Leve y Neiderhiser, 2013).
Al evaluar este referente metodológico en los estudios revisados para el presente documento, no se encuentran intervenciones que aborden la problemática del estrés laboral de forma holística, tal como lo explicita el modelo PPCT. Lo más cercano a un abordaje metodológico de este tipo son las intervenciones que combinan acciones orientadas a los individuos y acciones hacia la organización (Andersson-Felé, 2005; Brough y O’Driscoll, 2010; Hatinen et al., 2007; Le Blanc et al., 2007; Lingard, Francis y Turner, 2012).

Los componentes del modelo PPCT deben entenderse dentro de un sistema ecológico, holístico e interaccional. De esta premisa se destaca la noción de interdependencia, que se refleja en el concepto de los sistemas anidados (Guhn y Goelman, 2011), esto es, un sistema que contiene otro sistema como un conjunto de estructuras seriadas.

En este orden de ideas, Bronfenbrenner (2001) afirma que el sistema más interno es el microsistema, que es el nivel más cercano al sujeto e incluye los comportamientos, roles y relaciones interpersonales que experimenta el sujeto en sus contextos cotidianos más inmediatos. En el caso del trabajador, el microsistema está determinado por comportamientos, roles y relaciones propias de su contexto cotidiano, como familiares, amigos, pareja, hijos, compañeros de trabajo y jefes mediatos e inmediatos. En su mayoría, las intervenciones en el estrés laboral se instrumentan en el nivel de los comportamientos y los roles de la persona en su entorno laboral (Brinkborg et al., 2011; Hahn et al., 2011; Isaksson Ro et al., 2010; Orly et al., 2012; Unsworth y Mason, 2012; Wolever et al., 2012). En otros casos, en el diseño y ejecución de las intervenciones se ha considerado no solamente el patrón comportamental del trabajador, sino también la relación del individuo con su entorno laboral (e.g. características del trabajo), con los jefes o supervisores y con sus compañeros de trabajo (Hatinen et al., 2007; Le Blanc et al., 2007; Rickard et al., 2012). De acuerdo con Lamontagne et al. (2007), el número de esta clase de intervenciones va en aumento; en su revisión de los programas de intervención en el estrés laboral señalan que las intervenciones que combinan actividades sobre la organización y sobre el individuo se consideran de alta calidad porque muestran resultados favorables en el plano individual y en el organizacional. 
Según Bronfenbrenner (2001), el mesosistema señala las interrelaciones entre dos o más entornos en los que la persona participa activamente. Para el trabajador, el mesosistema está dado por la interacción entre los ambientes en los que se encuentra inmerso, como la relación bidireccional entre el trabajo, la familia y su vida social. En la actualidad adquiere cada vez más fuerza el realizar intervenciones orientadas a encontrar el balance entre el entorno familiar y el laboral (Brough y O’Driscoll, 2010; Lingard et al., 2012), pero no ocurre lo mismo cuando se trata de intervenciones que consideran la relación entre los entornos laboral y social del trabajador.

El exosistema comprende los ambientes que no involucran a la persona como un participante activo, pero en los que se producen hechos que afectan o se ven afectados por lo que sucede en el entorno en el cual se encuentra (Bronfenbrenner, 2001). En este artículo no se documentan intervenciones sobre el estrés laboral que consideren contextos que afectan indirectamente al trabajador, tales como el lugar de trabajo, la familia y los amigos de la pareja, o las redes sociales y el entorno escolar de los hijos, a pesar de que estos contextos también pueden ser fuente de estrés para el trabajador. En este nivel también se encuentra el contexto organizacional en las que se producen situaciones que afectan al trabajador de manera indirecta, como las fusiones empresariales, los cambios en la alta gerencia de las compañías o las decisiones en su manejo económico que afectan el presupuesto destinado a mantener o promover la salud laboral. Este artículo no aborda las intervenciones destinadas al manejo del estrés que puedan producir estos eventos.

El macrosistema hace referencia a la consistencia cultural, ideológica o de creencias que debe haber en los sistemas de orden menor (microsistema, mesosistema y exosistema) (Bronfenbrenner, 2001). Es decir, las características de la cultura, la religión, la etnia y el momento histórico-social en el que se encuentra la persona influyen en todos los niveles de su ambiente ecológico. Hoy día, en las intervenciones en el estrés laboral se reconoce la importancia de incluir en su diseño y puesta en práctica ciertos factores de este orden, como por ejemplo las políticas y legislación en salud laboral del país o de la región (Guthrie, Ciccarelli y
Babic, 2010; Leka, Jain, Iavicoli, Vartia y Ertel, 2011; Mellor et al., 2011).

El ambiente ecológico también presenta una dimensión temporal, que se describe con el término cronosistema. El cronosistema hace posible examinar la influencia de los cambios y las continuidades que se producen a través del tiempo en los ambientes en los que la persona vive. Los efectos de los procesos proximales pueden depender de cuándo y en qué orden suceden en la vida de una persona, así como cuándo se producen dentro del contexto histórico. Tal como lo afirma Bronfenbrenner (2001), el elemento tiempo tiene especial importancia en los diseños de investigación que utiliza el modelo bioecológico, dado que para demostrar que el desarrollo ha ocurrido, los elementos en el diseño y su relación dinámica deben haber influido en las características biopsicológicas de la persona en desarrollo durante un periodo prolongado.

En las intervenciones en el estrés laboral se ha ignorado dicha dimensión temporal en la mayoría de los casos, aunque se han encontrado efectos positivos de las intervenciones en la disminución del estrés mediante diseños cuasiexperimentales y mediciones postratamiento de corto y mediano plazo (Hahn et al., 2011; Le Blanc et al., 2007; Orly et al., 2012; Unsworth y Mason, 2012). Como afirma Landsbergis (2008), "muy pocos de estos estudios [y] programas de manejo del estrés [...] tienen un seguimiento a largo plazo, por lo que no sabemos si esas mejoras se mantienen en el tiempo" (p. 36). En consecuencia, los diseños cuasiexperimentales se ven limitados cuando se trata de explicar la efectividad de las intervenciones en el largo plazo. En este caso, los diseños longitudinales son los que consideran la relación dinámica que debe haber entre las diferentes variables que componen el diseño y los que aportan más precisión en relación con los determinantes y las consecuencias de las intervenciones.

Nielsen, Taris y Cox (2010b) sugieren el uso de métodos mixtos de investigación para mejorar la efectividad de las intervenciones. Estos diseños permiten agregar un componente cualitativo a un proceso netamente cuantitativo, o combinar aproximaciones cualitativas y cuantitativas a través de todas las fases de la intervención, y de esta manera incorporar las fortalezas de ambas metodologías 
para obtener resultados más robustos. Por ello, es cada vez más frecuente encontrar que los investigadores hacen uso de estrategias cuantitativas en combinación con las cualitativas y que implican la participación de diferentes grupos de interés de la organización (e.g. empleados, supervisores, gerentes) en el diseño e implementación de las intervenciones en el estrés laboral (Hatinen et al., 2007; Le Blanc et al., 2007; Lingard et al., 2012).

\section{Intervenciones en estrés laboral: elementos del modelo bioecológico para su abordaje}

La visión multisistémica del modelo bioecológico ofrece un marco explicativo con el cual las intervenciones en el estrés laboral pueden desarrollar- se porque extiende los focos de intervención más allá de los niveles individual y organizacional y hace posible generar estrategias de intervención de mayor alcance. Se espera entonces que los resultados de las intervenciones en el estrés laboral resulten de las interacciones entre las diferentes variables que componen el modelo metodológico PPCT dentro y a través de cada uno de estos sistemas.

Considerando los supuestos del modelo bioecológico, una propuesta para abordar las referidas intervenciones debe enfocarse en los factores de riesgo que surgen en los múltiples contextos sociales, desde los más próximos hasta los más lejanos al trabajador, lo que requiere articular las acciones preventivas en cada uno de los niveles de intervención (Figura 1).

Figura 1. Modelo bioecológico aplicado a las intervenciones en estrés laboral.

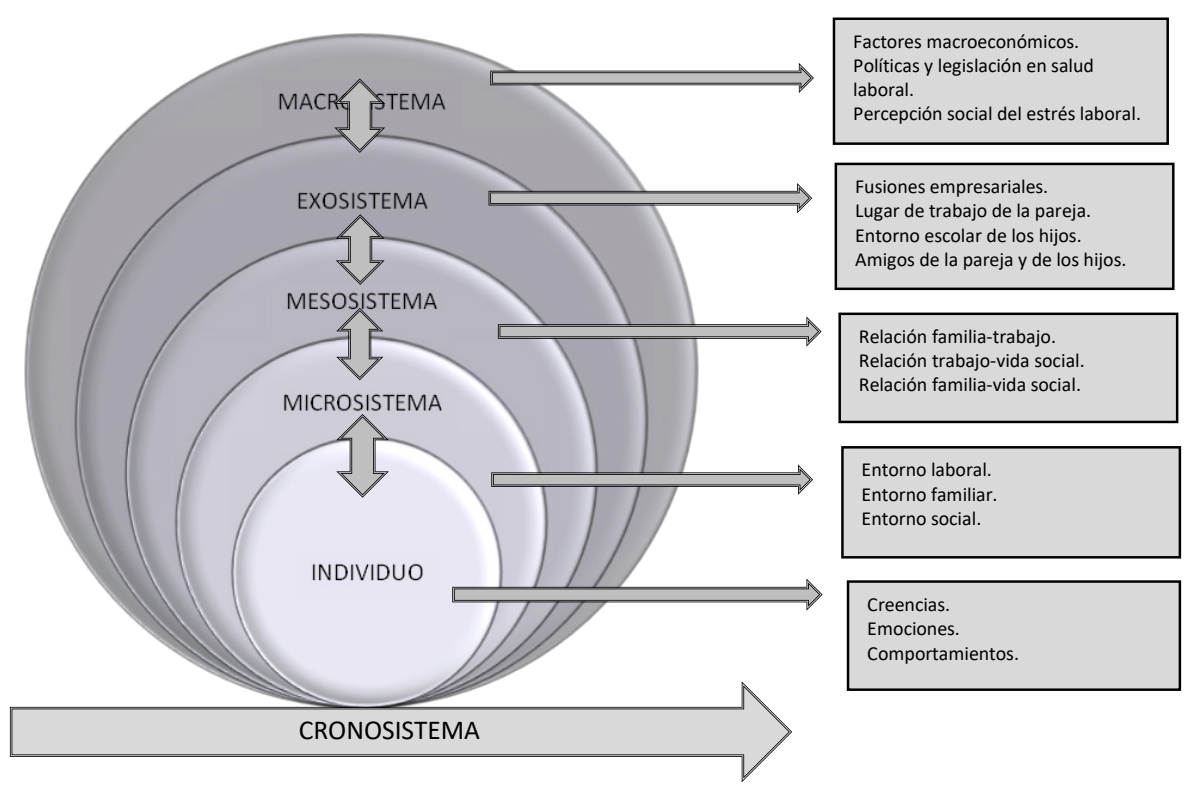

En el nivel del microsistema, se busca actuar sobre los patrones de comportamiento, hábitos, actitudes y creencias del trabajador, ya sea para fortalecer sus recursos individuales y prevenir el surgimiento del estrés (intervención primaria), para prepararlo para que desarrolle su capacidad de afrontamiento ante las condiciones estresantes (intervención secundaria), o para tratarlo y rehabilitarlo cuando sufra enfermedades o síntomas relacionados con el estrés (intervención terciaria). Diferentes autores arrojan evidencia empírica sobre estos focos de intervención (Brinkborg et al., 2011; Hahn et al., 2011; Isaksson et al., 2010; Orly et al., 2012; Unsworth y Mason, 2012; Wolever et al., 2012).
En este mismo nivel, el ámbito laboral es uno de los microsistemas más importantes para la intervención primaria, que es de orden preventivo y proactivo; así, las estrategias de intervención se orientan a reducir los estresores en el trabajo o a alterar las fuentes de estrés antes de que el trabajador lo experimente. Algunos ejemplos de acciones preventivas en este microsistema se relacionan con el cambio en las condiciones del trabajo, como su rediseño, la reducción de la carga laboral o la conformación de grupos colaborativos que incluyen a compañeros de trabajo o jefes inmediatos, entre muchos otros (Hatinen et al., 2007; Ipsen y Jensen, 2012; Le Blanc et al., 2007; Rickard et al., 2012). 
El contexto familiar es otro microsistema que tiene gran influencia sobre el trabajador porque se producen transferencias del trabajo a la familia y viceversa, las que pueden ser positivas o negativas. Por ello, las estrategias de intervención han sido de orden relacional y se ubican en el nivel del mesosistema, dando lugar a las intervenciones denominadas "balance familia-trabajo" (Brough y O’Driscoll, 2010; Lingard et al., 2012). Un plano desatendido en las intervenciones en el estrés laboral es la vida social del trabajador fuera de su entorno laboral y familiar. Las acciones preventivas en el mesosistema que es la relación trabajo-vida social pueden orientarse a identificar y fortalecer las redes de apoyo social con las que cuenta el trabajador (v.g. amigos, asociaciones, clubes deportivos). Por otra parte, en el exosistema del trabajador las intervenciones deben considerar, entre otros, factores tales como el entorno escolar de los hijos, el lugar de trabajo de la pareja y los cambios en el entorno organizacional, toda vez que pueden desencadenar respuestas de estrés en el trabajador.

El macrosistema es crucial para operar sobre las condiciones de orden social, político, económico y cultural. En el nivel macro, las intervenciones se han relacionado con la política, la legislación y las normas relativas a la salud laboral que existen en la región o en el país. Estas iniciativas han tenido un impacto favorable en el manejo del riesgo psicosocial asociado al estrés laboral (Guthrie et al., 2010; Leka et al., 2011; Mellor et al., 2011); sin embargo, es necesario que en esas intervenciones participen todos los grupos de interés (Estado, empresa, directivos y trabajadores) para asegurar la existencia de esas políticas y su traducción en prácticas efectivas en la organización. Guthrie et al. (2010) afirma que la salud y el bienestar de los trabajadores pueden promoverse mediante la legislación, las acciones conjuntas entre todos los grupos de interés y los incentivos financieros en las organizaciones.

Conjugando factores del exosistema y del macrosistema en el diseño, implementación y evaluación de las intervenciones en estrés laboral, los elementos a considerar serian numerosos; por ejemplo, la alineación de la estrategia organizacional en gestión financiera y presupuestal con la estrategia en gestión humana y salud laboral; los valores y la cultura de la organización; las políticas institucionales que se traduzcan en diagnósticos y programas de intervención relacionados con la salud y el bienestar de los trabajadores; la normativa estatal que impulse el desarrollo de este tipo de programas en las empresas, y las políticas gubernamentales y las directrices económicas en materia de salud laboral en el país, entre muchos otros elementos.

En resumen, el modelo bioecológico aplicado a las intervenciones en el estrés laboral involucra al trabajador, las relaciones que el trabajador establece con otros en su entorno laboral, familiar y social, y las relaciones interinstitucionales con las entidades que diseñan la política pública en el área de la salud laboral y que establecen las directrices en materia de salud en el trabajo. A todos estos elementos se suma la dimensión temporal, que atraviesa todos los sistemas y que implica el efecto del tiempo en el curso de la vida de un trabajador, así como los cambios y continuidades presentes en los diferentes contextos con los que se relaciona y el momento histórico en el cual se encuentre. Como lo sugieren Hermosa y Perilla (2015), el diseño y puesta en marcha de las intervenciones en el estrés laboral requieren el acercamiento multinivel que, como se expuso previamente, ofrece el modelo bioecológico.

\section{CONCLUSIONES}

El propósito central de este artículo fue analizar las intervenciones en el estrés laboral a la luz de los fundamentos conceptuales y metodológicos del modelo bioecológico de Bronfenbrenner. Para tal efecto, se profundizó en la perspectiva teórico-conceptual y en los aspectos metodológicos del modelo, y paralelamente se analizaron los programas de intervención basados en dicha aproximación.

La revisión de la literatura evidencia que esos programas de intervención se han centrado primordialmente en el individuo, y aunque han demostrado ser efectivas para éste, no lo son necesariamente para la organización. Por ello, las intervenciones que combinan acciones centradas en el individuo y en la organización muestran una tendencia creciente. Así mismo, los diseños (cuasi) experimentales son los más utilizados en las intervenciones en estrés laboral aunque su efectividad 
a largo plazo ha sido cuestionada, de tal manera que se sugiere el uso de métodos mixtos como medida para incrementar la efectividad de las intervenciones. Sin embargo, más allá de combinar estrategias metodológicas de orden cuantitativo y cualitativo, los factores macroorganizacionales (v.g. fusiones, cambios en infraestructura física o tecnológica o movimientos sindicales) también dificultan la efectividad de las intervenciones desde el diseño, implementación y evaluación.

En cuanto al modelo de Bronfenbrenner, dos elementos son centrales, el ambiente ecológico, entendido como un conjunto de estructuras seriadas interdependientes en las que un sistema contiene otro, y el modelo PPCT como un diseño de investigación que hace posible estudiar simultáneamente los procesos proximales, las características de la persona y del contexto y las relacionadas con el tiempo. Sin duda, abordar los problemas en el estrés laboral con una visión holística e interaccional es un aporte incuestionable a la investigación de este tipo de intervenciones y obliga a repensar las estrategias metodológicas que se han utilizado tradicionalmente en este campo. El mismo Bronfenbrenner afirma que el modelo PPCT es un diseño de investigación complejo por las múltiples variables que deben considerarse; pese a ello, las intervenciones requieren de una perspectiva integral y dinámica porque las acciones del trabajador y sus problemas de salud no aparecen aisladas, sino que afectan y son afectadas por las relaciones que el individuo establece con sus entornos próximos y distantes.

En este orden de ideas, se ha esbozado una propuesta para abordar esas intervenciones asumiendo las dimensiones consideradas en el modelo bioecológico de Urie Bronfenbrenner. Dicha propuesta establece que en cada uno de los contextos sociales con los que se relaciona el trabajador hay diversos factores probables de riesgo como fuentes de estrés, y que las estrategias preventivas deben articularse en cada uno de los niveles de intervención; es decir, las intervenciones en el estrés laboral deben considerar al trabajador como una persona en continuo ajuste a sus entornos inmediatos (laboral, familiar y social), que también son dinámicos y cambiantes. A su vez, estos entornos se relacionan con contextos más distantes del trabajador pero que lo afectan de manera indirecta (cambios macroorganizacionales, políticas públicas, aspectos macroeconómicos y demás). En otras palabras, las intervenciones deben orientarse partiendo de una perspectiva holística que entienda el individuo en función de su ambiente organizacional y social. Tomar en cuenta estas dimensiones facilitará al investigador responder a las preguntas sobre qué intervenciones funcionan, para quién y en qué circunstancias.

\section{REFERENCIAS}

Andersson-Felé L. (2005). Age related work load-a work environment intervention with a life course perspective. International Congress Series, 1280, 341-346. Disponible en https://doi.org/10.1016/j.ics.2005.01.017.

Brinkborg, H., Michanek, J., Hesser, H. y Berglund, G. (2011). Acceptance and commitment therapy for the treatment of stress among social workers: A randomized controlled trial. Behavior Research \& Therapy, 49, 389-398.

Bronfenbrenner, U. (2001). The bioecological theory of human development. En N. J. Smelser y P. B. Baltes (Eds.): International Encyclopedia of the Social and Behavioral Sciences. (v. 10, pp. 6963-6970). New York: Elsevier.

Bronfenbrenner, U. y Morris, P. (2006). The bioecological model of human development. En W. Damon y R. M. Lerner (Eds.): Handbook of Child Development. (v. 1, pp. 793-828). Hoboken, NJ: Wiley.

Brough, P. y O’Driscoll, M. (2010). Organizational interventions for balancing work and home demands: an overview. Work \& Stress, 24(3), 280-297. doi: 10.1080/02678373.2010.506808.

Brough, P. y O’Driscoll, M. (2005). Work-family conflict and stress. En A. S. Antoniou y C. L. Cooper (Eds.): Research companion to organizational health psychology. (pp. 346-365). London: Edward Elgar Publishing.

Cox, T., Taris, T.W. y Nielsen, K. (2010). Organizational interventions: Issues and challenges. Work y Stress, 24 (3), 217-218. doi: 10.1080/02678373.2010.519496.

Glanz, K., Rimer, B. y Viswanath, K. (2008). Theory, research, and practice in health behavior and health education. En K. Glanz, B. Rimer y K. Viswanath (Eds.), Health Behavior and Health Education. Theory, Research, and Practice. (pp. 23-40). USA: Wiley. 
Guhn, M. y Goelman, H. (2011). Bioecological theory, early child development and the validation of the population-level early development instrument. Social Indicators Research, 103, 193-217. doi: 10.1007/s11205-011-9842-5.

Guthrie, R., Ciccarelli, M. y Babic, A. (2010). Work-related stress in Australia: The effects of legislative interventions and the cost of treatment. International Journal of Law Psychiatry, 33(2), 101-115.

Hahn, V., Binnewies, C., Sonnentag, S. y Mojza, E. (2011). Learning how to recover from job stress: Effects of a recovery training program on recovery, recovery-related self-efficacy, and well-being. Journal of Occupational Health Psychology, 16(2), 202-216. doi: 10.1037/a0022169.

Hansson, A.S., Vingard, E., Arnetz, B.B. y Anderzén, I. (2008). Organizational change, health, and sick leave among health care employees: A longitudinal study measuring stress markers, individual, and work site factors. Work \& Stress, 22(1), 69-80. doi: $10.1080 / 02678370801996236$.

Hatinen, M., Kinnunen, U., Pekkonen, M. y Kalimo, R. (2007). Comparing two burnout interventions: Perceived job control mediates decreases in burnout. International Journal of Stress Management, 14(3), 227-248. doi: 10.1037/1072-5245.14.3.227.

Henry, J. (2005). The healthy organization. En A. S. Antoniou y C. L. Cooper (Eds.): Research companion to Organizational Health Psychology (pp. 382-392). London: Edward Elgar Publishing.

Hermosa, A.M. y Perilla, L.E. (2015). Retos investigativos en psicología de la salud ocupacional: el estrés laboral. Revista de la Facultad Nacional de Salud Pública, 33(2), 252-261. doi: 10.17533/udea.rfnsp.v33n2a12.

Ipsen, C. y Jensen, P.L. (2012). Organizational options for preventing work-related stress in knowledge work. Internatioal Journal of Industrial Ergonomics, 42(4), 325-334.

Isaksson Ro, K.E., Tyssen, R., Hoffart, A., Sexton, H., Aasland, O.G. y Gude, T. (2010). Aethree-year cohort study of the relationships between coping, job stress and burnout after a counselling intervention for help-seeking physicians. BMC Public Health, 10, 2-13.

Kortum, E., Leka, S. y Cox, T. (2010). Psychosocial risks and work-related stress in developing countries: Health impact, priorities, barriers and solutions. The International Journal of Occupational Medicine and Environmental Health, 23(3), 225-238.

Lamontagne, A., Keegel, T., Louie, A., Ostry, A. y Landsbergis, P. (2007). A systematic review of the job-stress intervention evaluation literature, 1990-2005. The International Journal of Occupational Medicine and Environmental Health, 19(13), 268-280.

Landsbergis, P. (2008). ¿Qué se debe hacer para prevenir los riesgos psicosociales en el trabajo? Archivos de Prevención de Riesgos Laborales, 11(1), 36-44.

Le Blanc, P., Hox, J., Schaufeli, W., Taris, T. y Peeters, M. (2007). Take care! The evaluation of a team-based burnout intervention program for oncology care providers. Journal of Applied Psychology, 92(1), 213-227. doi: 10.1037/0021-9010.92.1.213.

Leka, S., Jain, A., Iavicoli, S., Vartia, M. y Ertel, M. (2011). The role of policy for the management of psychosocial risks at the workplace in the European Union. Safety Science, 49, 558-564. doi: 10.1016/j.ssci.2010.02.002.

Lingard, H., Francis, V. y Turner, M. (2012). Work-life strategies in the Australian construction industry: Implementation issues in a dynamic project-based work environment. International Journal of Projessional Management, 30, 282-295. doi: 10.1016/j.ijproman.2011.08.002.

Maes, S. y Boersma, S.N. (2005). Applications in health psychology: How effective are interventions? En S. Sutton, A. Baum y M. Johnston, M. (Eds.): The SAGE Handbook of Health Psychology (pp. 299-325). London: Sage.

Mellor, N., Mackay, C., Packham, C., Jones, R., Palferman, D., Webster, S. y Kelly, P. (2011). 'Management standards' and work-related stress in Great Britain: Progress on their implementation. Safety Science, 49, 1040-1046. doi: 10.1016/j. ssci.2011.01.010.

Ministerio de la Protección Social de la República de Colombia (2007). Primera Encuesta Nacional de Condiciones de Salud y Trabajo en el Sistema General de Riesgos Profesionales. Bogotá: Autor. Disponible en www.minsalud.gov.co/Documentos\%20y\%20Publicaciones/ENCUESTA\%20SALUD\%20RP.pdf.

Ministerio del Trabajo de la República de Colombia (2015). Segunda Encuesta Nacional de Condiciones de Seguridad y Salud en el Trabajo en el Sistema General de Riesgos Laborales. Bogotá: Autor. Disponible en http://fondoriesgoslaborales.gov.co/ documents/publicaciones/encuestas/II_ENCUESTA_NACIONAL_CONDICIONES_SST_COLOMBIA_2013.pdf.

Nielsen, K., Randall, R., Holten, A. y Rial-Gonzalez, E. (2010a). Conducting organizational-level occupational health interventions: What works? Work \& Stress, 24(3), 234-259. doi: 10.1080/02678373.2010.515393.

Nielsen, K., Taris, T.W. y Cox, T. (2010b). The future of organizational interventions: Addressing the challenges of today's organizations. Work \& Stress, 24(3), 219-233. doi: 10.1080/02678373.2010.519176.

Organización Internacional del Trabajo (ОIт) (2010). Riesgos emergentes y nuevos modelos de prevención en un mundo de trabajo en transformación. Ginebra: OIT.

Organización Internacional del Trabajo (OIT) (2016). Estrés en el trabajo. Un reto colectivo. Turín (Italia): Centro Internacional de Formación de la OIT. 
Orleans, T. (2008). Foreword. En K. Glanz, B. Rimer y K. Viswanath (Eds.): Health behavior and health education. Theory, research, and practice. New York: Wiley.

Orly, S., Rivka, B., Rivka, E. y Dorit, S.E. (2012). Are cognitive-behavioral interventions effective in reducing occupational stress among nurses? Applied Nursing Research, 25, 152-157. doi: 10.1016/j.apnr.2011.01.004.

Reiss, D., Leve, L. y Neiderhiser, J. (2013). How genes and the social environment moderate each other. American Journal of Public Health, 103, 111-121. doi: 10.2105/AJPH.2013.301408.

Rickard, G., Lenthall, S., Dollard, M., Opie, T., Knight, S., Dunn, S., Wakerman, J., MacLeod, M., Jo Seiler, J. y Brewster-Webb, D. (2012). Organizational intervention to reduce occupational stress and turnover in hospital's nurses in the Northern Territory, Australia. Collegian, 19(4), 211-221.

Unsworth, K. y Mason, C. (2012). Help yourself: The mechanisms through which a self-leadership intervention influences strain. Journal of Occupational Health Psychology, 17(2), 235-245. doi: 10.1037/a0026857.

Wolever, R., Bobinet, K., McCabe, K., Mackenzie, E., Fekete, E., Kusnick, C. y Baime, M. (2012). Effective and viable mindbody stress reduction in the workplace: A randomized controlled trial. Journal of Occupational Health Psychology, 17(2), 246-258. doi: 10.1037/a0027278. 